\title{
Proceeding
}

Supplementary Issue: Spring Conferences of Sports Science. Costa Blanca Sports Science Events, 19-20 June 2020. Alicante, Spain.

\section{Application of isocapnic hyperpnea in the treatment of vertebral column paramorphisms: Clinical case reports}

\author{
GIUSEPPE DI FABRIZIO1 1 , OMAR GAETANO MARIA MINGRINO², VINCENZO CRISTIAN \\ FRANCAVILLA ${ }^{2}$
}

${ }^{1}$ Voluntary researcher graduate in Physical Therapy, Italy

${ }^{2}$ School of Engineering, Architecture, and Motor Sciences, Kore University of Enna, Enna, Italy

\begin{abstract}
The aim of the study is to frame the use of Isocapnic Hyperpnea in the treatment of different spine paramorphisms through the use of SpiroTiger ${ }^{\odot}$. Several studies in recent years are increasingly looking for the current correlations between the respiratory musculature and the Fine Postural System. Based on this consideration, we decided to use respiratory exercise to induce a considerable modification of postural alignment. To provide meaningful data, we associated an objective baseline assessment with an objective assessment using spinometry. Four subjects are identified, undergoing an initial objective assessment of

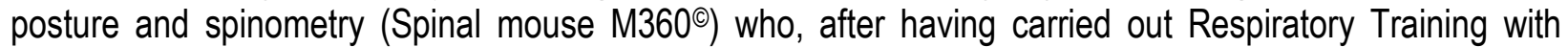
SpiroTiger ${ }^{\ominus}$ and postural gymnastics, were followed-up between the 5 th and 8 th sessions. The first two cases had postural alterations (lumbar hyperlordosis and dorsal cyphosis) while the assessment of the other subjects showed no postural deficits in the upright station. However, during anterior bending and/or the extension of the trunk in maximum inhalation, there were joint limitations that reduced the Range of Motion (ROM). Although the subjects examined had different Paramorphisms, the final assessment shows in all cases an increase in the ROM of the spine (bending $=6-12^{\circ}$ extension $=2^{\circ}$ ), better postural alignment resulting in a reduction in algies.
\end{abstract}

Keywords: Posture; Respiratory Training; Isocapnic Hyperpnea.

\section{Cite this article as:}

Di Fabrizio, G., Mingrino, O.G.M. \& Francavilla, V.C. (2020). Application of isocapnic hyperpnea in the treatment of vertebral column paramorphisms: Clinical case reports. Journal of Human Sport and Exercise, 15(3proc), S504-S517. doi:https://doi.org/10.14198/hhse.2020.15.Proc3.04

Corresponding author. Voluntary researcher graduate in Physical Therapy, Italy.

E-mail: difa97@outlook.it

Supplementary Issue: Spring Conferences of Sports Science. Costa Blanca Sports Science Events, 19-20 June 2020. Alicante, Spain.

JOURNAL OF HUMAN SPORT \& EXERCISE ISSN 1988-5202

(c) Faculty of Education. University of Alicante

doi:10.14198/jhse.2020.15.Proc3.04 


\section{INTRODUCTION}

Motor activity is a complex phenomenon within the social context and characterizes the culture, tradition and civilization of a people. The moderate technology and the great innovations applied to sport have significantly carried out all the social inclusion beyond the physical of disabled people. The fundamental role that physicalsporting activity plays in promoting positive life that is able to favour "greeting", understood in a general sense and not as a simple "absence of disease", is recognized. The National Health Plan contains innovative and highly interesting aspects such as to build a real dimension of the concept of prevention. (Francavilla et al, 2007).

In fact, it becomes aware of the social and educational value of motor activity which is capable of enhancing man's "needs" as a playful and moving activity and as socializing techniques to discover, know and live in harmony with his Body. In the healthy population, the prevention of diseases and the maintenance of the state of health are based on the identification and control of both risk factors and incorrect errors that favour the onset of different pathologies; not least the identification and control of harmful factors harmful to health. (Francavilla \& Francavilla, 2013).

Various studies now define the high level of interaction that exists between the respiratory musculature and the correct maintenance of the posture.

Kim MS et al. (2017) demonstrated the correlation between Cranial Angle and Cranial Rotation Angle and Respiratory Functions in patients with Forward Head Postures. In addition, the Cranial Angle and Cranial Rotation Angle would affect the tone and strength developed by scalene muscles and Sternocleidomastoid muscle. From this correlation has been drawn the conclusion that the maintenance of a correct posture of the neck can increase respiratory functions.

Szopa A. et al .(2017) proved that on subjects with Mild Idiopathic Scoliosis there are no alterations in respiratory functions, however the respiratory performance is affected in kyphotic subjects.

The Respiratory Gymnastics, understood as elongation and reinforcement of the respiratory musculature, with different techniques including the isocapnic hyperpnea, has long been widely applied in the field of chronic respiratory diseases. (Göhl et al, 2016).

The production of reactive oxygen and RNS is a fundamental feature of mammalian physiology, cellular respiration and cell signalling, and essential for muscle function and training adaptation. (Francavilla et al, 2018).

In addition, the same pathologies of the respiratory system induce defence attitudes (e.g. dorsal kyphosis or anterocollis), characterized by the retraction of the inhalation musculature and weakening of the exhalation, which further reduce respiratory performance.

Painful contractures and a reduction in the range of movement are frequently associated with rigid collagen tissue inside and around the skeletal muscle, as well as with other connective tissues used in the transmission of force; the fascial function, such as for example that of the joint capsules, tendons or epimysium and endomysium, could be altered due to trauma and / or inflammation. (Francavilla et al, 2016). 
On the other hand, other studies define the importance of the diaphragm as modulator of muscle chains and stabilizer of the spine. (Kocjan et al, 2017; Bordoni \& Zanier, 2013; Bordoni et al, 2016; Hodges \& Gandevia, 2000; Hodges et al, 1997).

The Diaphragm, in addition to being a centre for the modulation of muscle chains, through its own fascial relationships and connections, is a fundamental crossroads of information that is continuously taken by the Fine Postural System.

Another factor that affects the spine is a good activation of the Core (Abdominal, Diaphragm, Peritoneum and Paravertebral) that regulates the Intrabdominal Pressure and cooperates with the stabilization of the spine during the movement.

Therefore, the correct mobility and tone of the diaphragm and of the inspiratory musculature, associated to the selective reinforcement of the deficit musculature, should constitute an ulterior instrument spendable in the within of the Postural Re-education.

\section{METHOD}

All subjects have been subjected to a first basic postural evaluation that includes: objective evaluation, lead wire measurement, Fukuta Test, Forward Bending Test and evaluation of the podalic support on the podoscope.

In order to optimize the evaluation of the rachis each subject was then subjected to Spinal Mouse M360@.

This device is equipped with two rollers embedded on a mobile support that allow the monitoring of the spiny contour. The contour is recorded by three sensors that through a 3D Bluetooth connection transmit the clinically relevant data to the program for processing.

The treatment is carried out through the use of SpiroTiger@, a device that exploiting the principle of Isocapnic Hyperpnea allows to work with a high Respiratory Frequency and high Respiratory Volumes without the risk of incurring symptoms related to Hyperpnea (Dizziness, headache, tingling and nausea). The apparatus is used at low respiratory frequency for the mobilization of the chest while with high frequencies it induces a reinforcement of the respiratory musculature. (Wirth et al, 2014, 2016; Włodarczyk \& Barinow-Wojewódzki, 2015).

All the subjects have been subordinated to intermediate appraisals and, at the end of the protocol, also to a final revaluation. Assuming the data we compared with the pre-treatment results.

\section{Clinical case report}

Below are reported 4 clinical cases with different paramorphisms subjected to customized Postural Reeducation protocols.

\section{First clinical case}

At the first evaluation, the patient presents lumbar hyperlordosis and hypomobility of the T9-T10-T11 vertebrae. The evaluation of the coronal plane shows that a scoliotic right concave lumbar attitude resulting in asymmetry, for tone and trophism, of the paravertebral muscles. (Figure 1). 


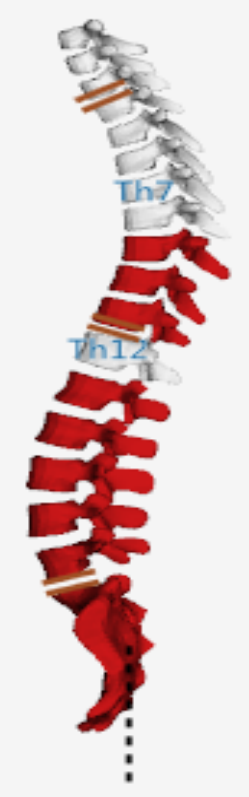

Figure 1. Pre-treatment spinometry.

In fact, the paravertebral musculature of left side was elongated and hypotonic while the abdominal and loins square of right side assumed a shortening position. The assessment of the podalic support shows a slight flatness of the left foot with displacement of the load on the metatarsal heads. The patient was subjected to 8 sessions in 4 weeks consisting of a first part of Global Stretching and selective reinforcement and a second part of Isocapnica Hyperpnea Training with SpiroTiger ${ }^{\odot}$. The personalized Respiratory Gymnastics protocol has been designed to reconcile the mobilization of the chest, with respiratory frequency sessions between 16 and 18 acts/min, and strengthening of the abdominal muscles with high respiratory frequency sessions (28-30 acts/min). Two intermediate evaluations are carried out with Spinal Mouse D360 ${ }^{\circ}$. After 4 sessions the subject presents an increase of the ROM in forward bending of $6^{\circ}$. (Figure 2).

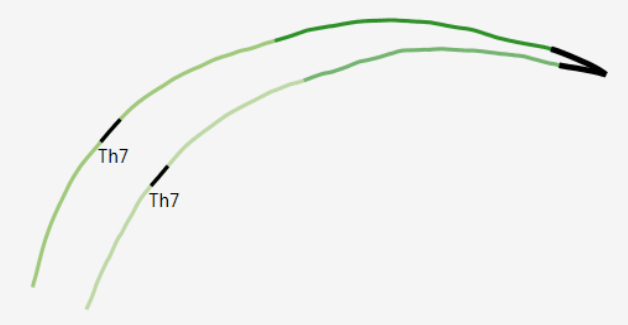

Figure 2. Forward bending ROM. 
At the seventh session the subject undergoes a re-entry of the positional range of the segments T3-T4, T5T6, L2-L3 and L4-L5. At the end of the 8 sessions there is an increase in the overall mobility of the column, but more indicative of the thoracic tract, a realignment of the pelvis and an improvement of the vertebral harmonization index. (Figures 3-4).

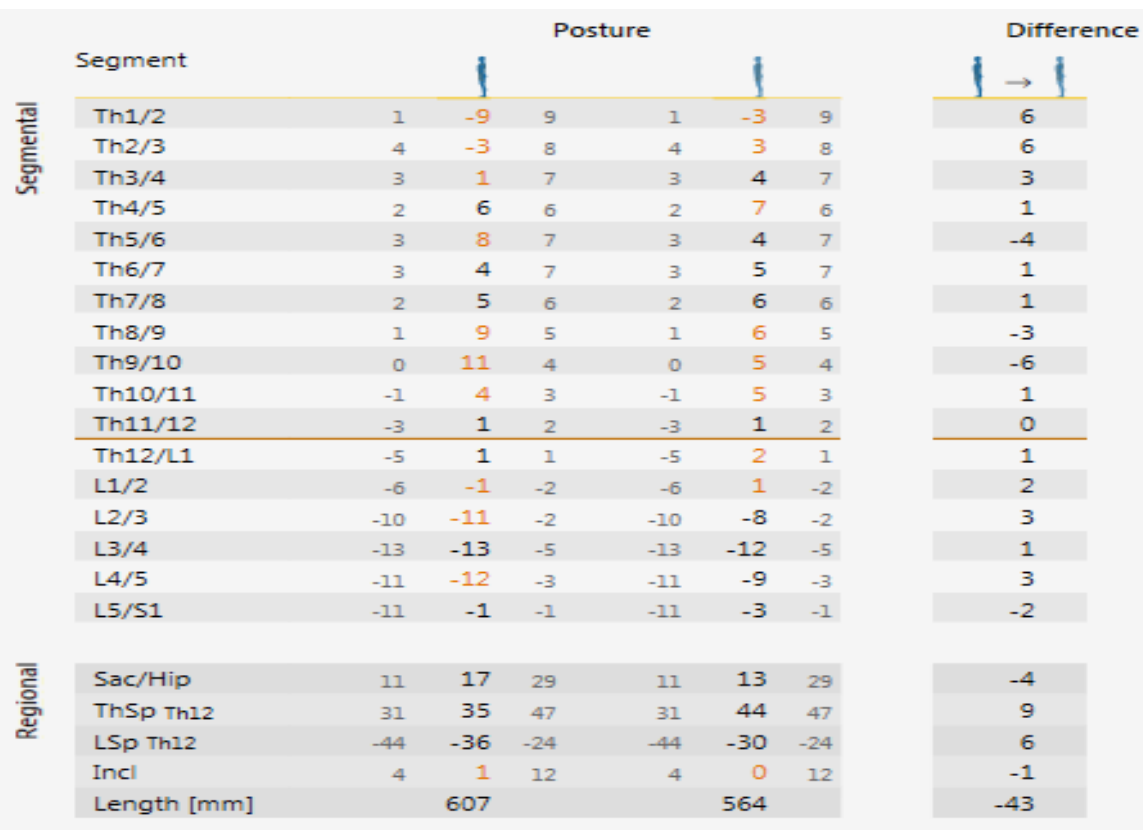

Figure 3. Pre-post treatment chart comparison.
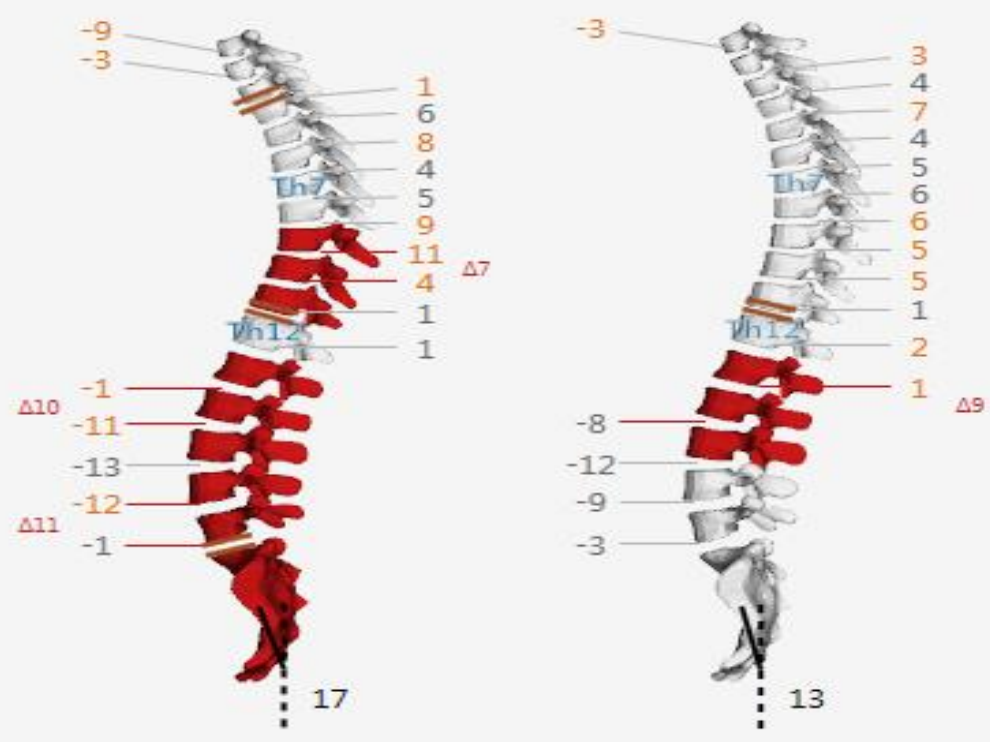

Figure 4. Pre-post treatment numeric comparison. 


\section{Second clinical case}

At the first evaluation the patient presented a slight dorsal kyphosis and a right inclination of the head resulting in retraction of right trapezius. The muscular retractions involved have determinate a primary hypomobility of the T5-T6-T7 dorsal vertebrae with lumbar compensation on L1-L2-L3. (Figure 5).

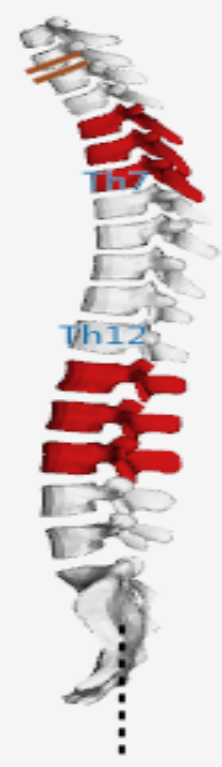

Figure 5. Pre-treatment Spinometry.

The evaluation of the podalic support did not report any significant changes. This postural condition induced interscapular back pain on the left side and cervical pain. The patient has been subjected to a cycle of 6 sessions in 3 weeks in which stretching exercises, neck and chest mobilization and strengthening exercises were combined.

In fact in a first phase the focus was on the stretching of the trapezius, the periscapular musculature and the lumbar rachis, attic mobility of the neck and shoulder and sessions of Respiratory Gymnastics with SpiroTiger@ at medium-low respiratory frequency $(16-18 \mathrm{acts} / \mathrm{min})$ to mobilize the chest and stretch the inspiratory muscles. In a later stage, isometric and eccentric reinforcement exercises were inserted to recondition the shoulder and dorsal muscles. The follow-up at the end of the sessions highlights the re-entry of negative parameters of mobility, with an increase of $12^{\circ}$ in forward bending, and of the positioning of the individual vertebral joints. (Figures 6-7-8-9).

In addition, the patient does not report any pre-existing pain at the first evaluation. 


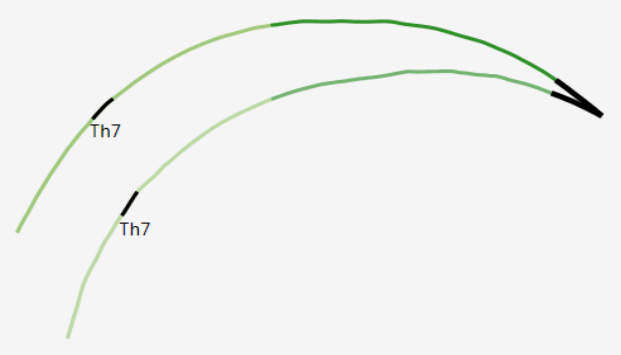

Figure 6. Forward bending ROM.

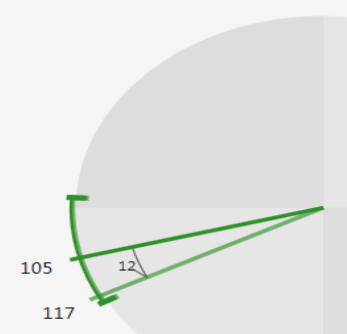

Figure 7. Forward bending ROM.

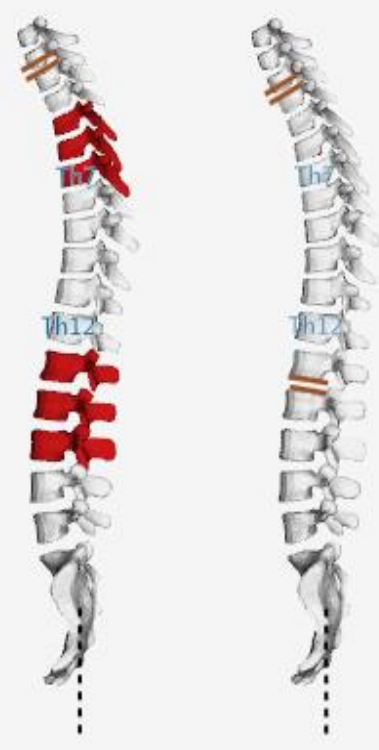

Figure 8. Pre-post treatment chart comparison. 


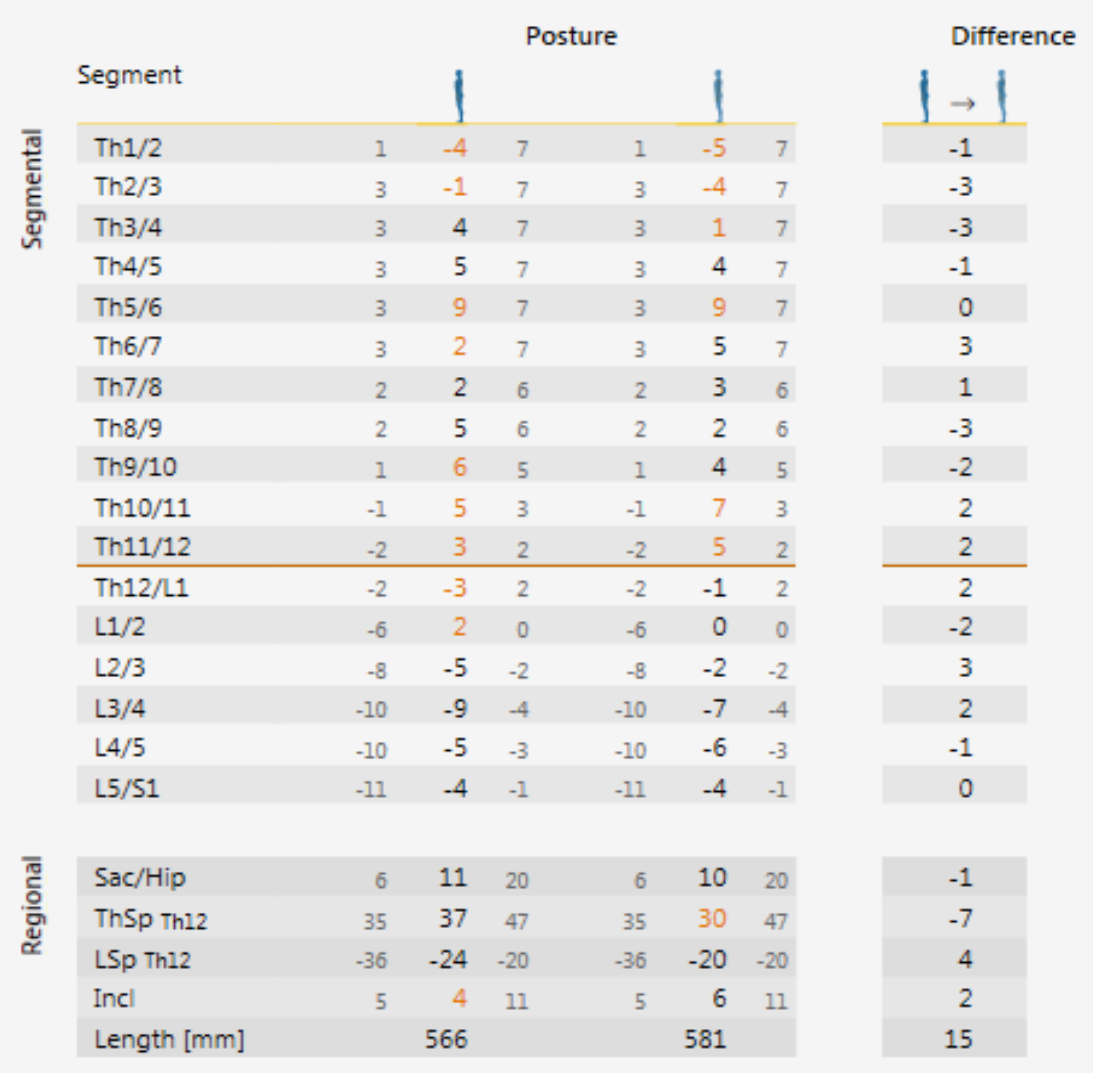

Figure 9. Pre-post treatment numeric comparison.

\section{Third clinical case}

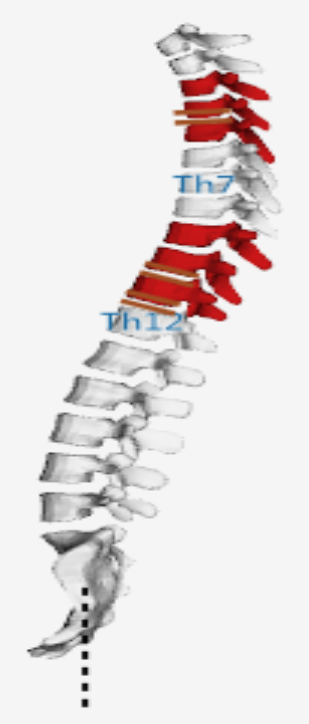

Figure 10. Pre-treatment spinometry. 
The patient did not present any relevant alterations after the objective evaluation. However, when carrying out the evaluation with Spinal Mouse D3600, it was highlighted a reduced mobility of the rachis and in forward bending and extension with maximum inspiration concentrated on the vertebrae T8-T9-T10. (Figure 10).

No pain was reported. In this case, 5 sessions were planned which were enough to achieve significant results. The aim of the protocol was to increase mobility in bending and extension of the torso and to improve the inhalation phase.

For this reason, the sessions were divided into a first phase of Respiratory Gymnastics with SpiroTiger@ and a second phase of stretching of the back muscle chain. Isocapnic hyperpnea work was carried out in the first sessions with a respiratory frequency of 14-22 acts/min to increase chest mobility, and in the subsequent sessions with high respiratory frequencies (27-30 acts/min) to strengthen and vascularize the inspiratory muscles.

At the end of the five sessions the subject has an increase in mobility of both the cervical spine, both the last thoracic vertebrae and the lumbosacral hinge. This result is further objected by the increase in the length of the spine in forward benging of $28 \mathrm{~mm}$.

Moreover, the best recruitment of inspiratory muscles allows to increase the extension of the trunk. (Figures 11-12).
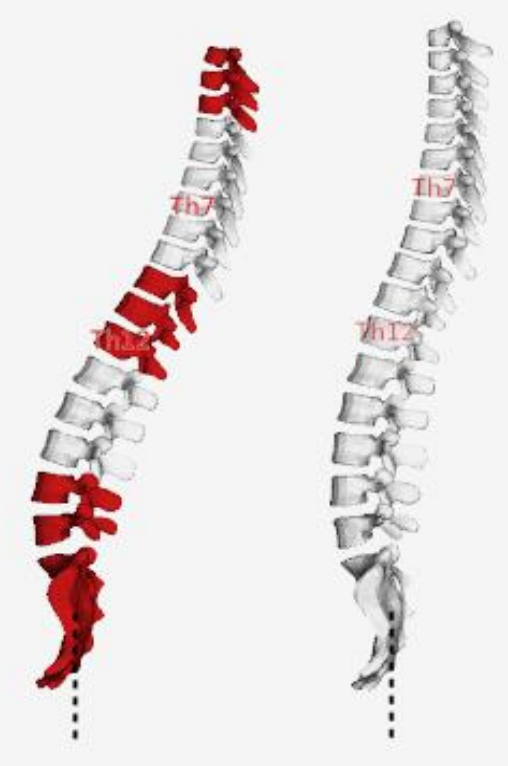

Figure 11. Pre-post treatment chart comparison. 


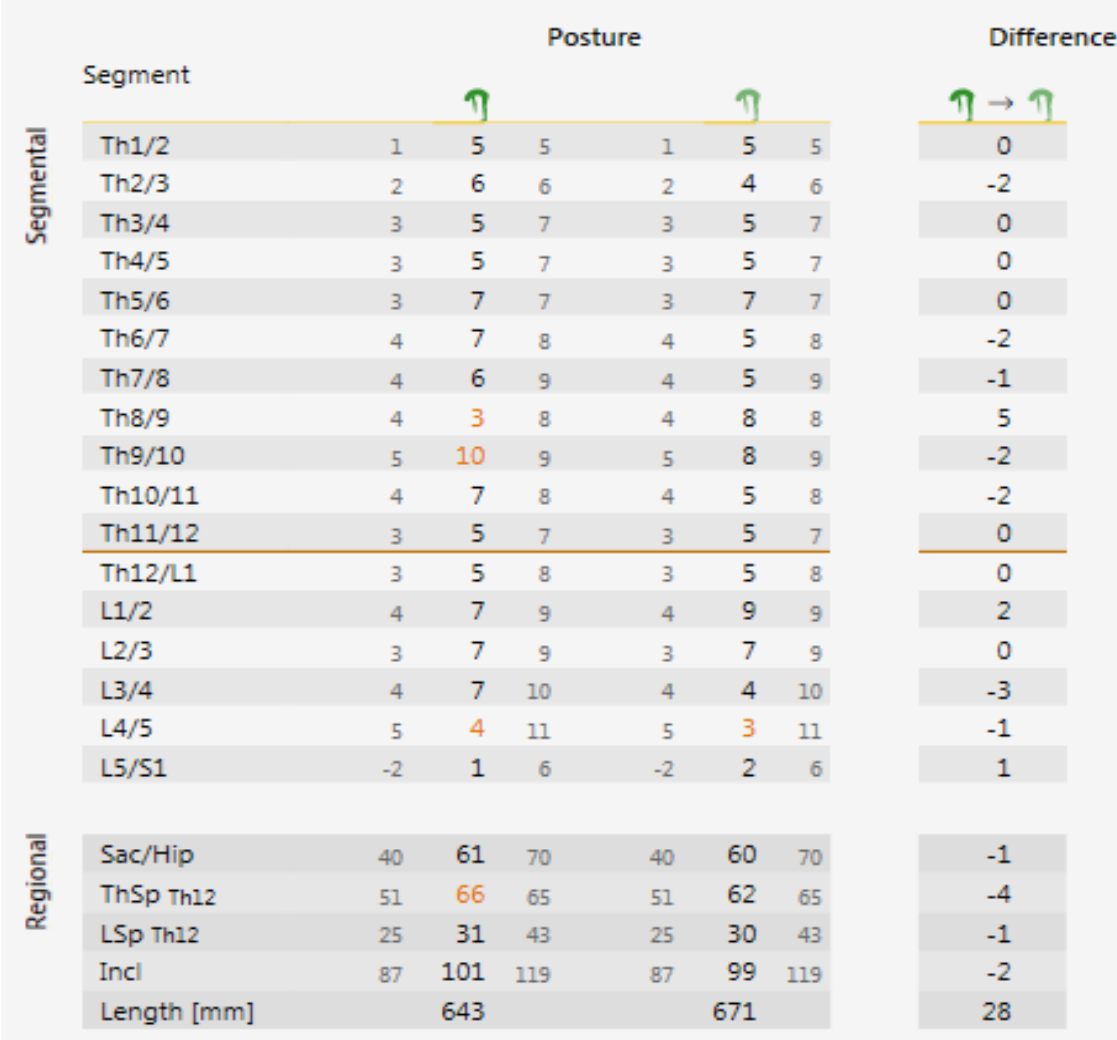

Figure 12. Pre-post treatment numeric comparison.

\section{Fourth clinical case}

The patient did not have any postural alterations either in standing position or in forward bending and there were no limitations in torso flexion, however, during maximum inhalation the Spinal Mouse D360@ detected significant joint limitations at the expense of T3-T4,T4-T5, T9-T10,T10-T11 and T11-T12.

Also, during maximum inhalation, the patient reports back pain.

He is subjected to a 6-session protocol with the aim of increasing the active ROM in torso extension and at the same time improving the recruitment of respiratory muscles. At the end of each session the patient is subjected to stretching of the back muscle chain.

In order to increase the ROM, the patient does Respiratory Gymnastics and in particular exercises of proprioception of the breath (focusing on respiration bases-thoracic and claveare). This work is first done on the back in supine position, in the following sessions the SpiroTiger@ is used in sessions with open eyes and closed eyes. Then the thorax is mobilized in isocapnic hyperpnea at 14-16 acts/min.

To strengthen the inspiratory muscles the patient is subjected to isocapnic hyperpnea training with incremental sessions with respiratory frequency of 22-24 acts/min.

At the end of the sessions the final evaluation highlights the resolution of the joint blocks from $\mathrm{T} 3$ to $\mathrm{T} 5$ and from T9 to T12 resulting in an increase in ROM $\left(2^{\circ}\right)$ and resolution of algic symptoms. (Figures 13-14-15). 


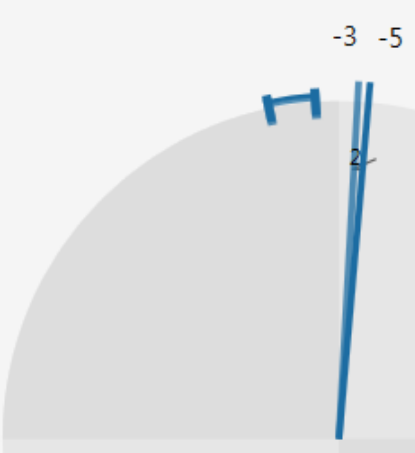

Figure 13. Torso extension in maximum inhalation ROM.

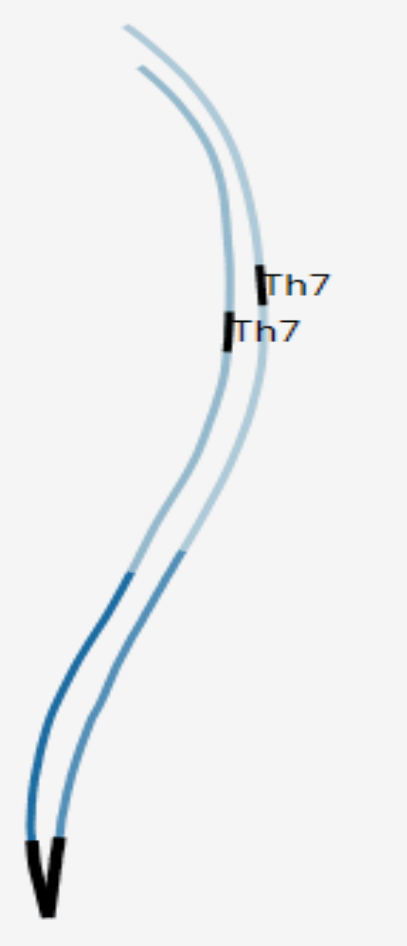

Figure 14. Torso extension in maximum inhalation ROM. 


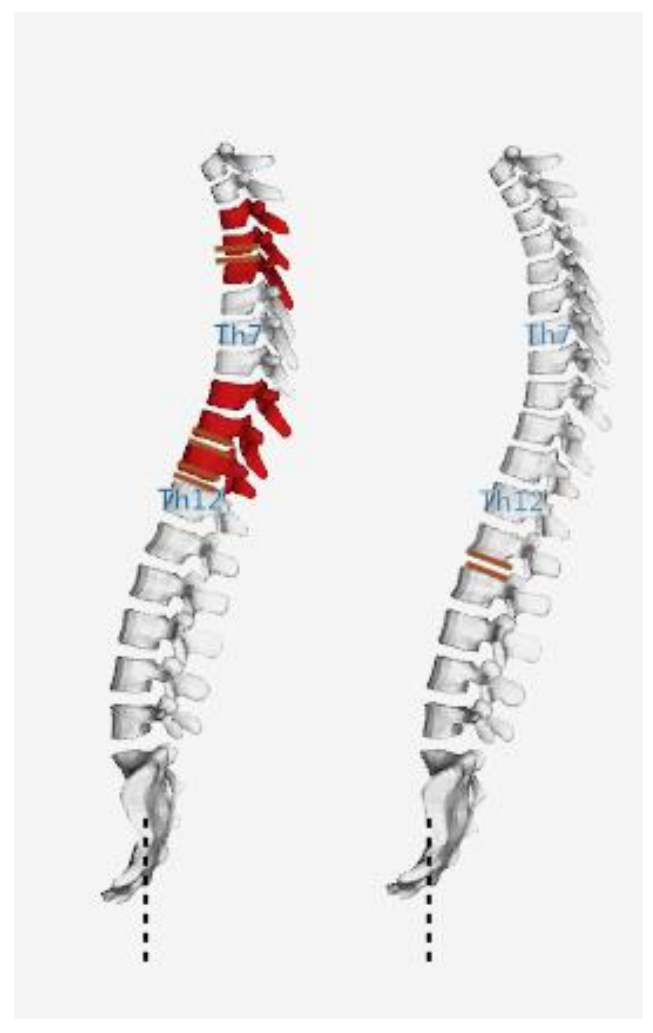

Figure 15. Pre-post treatment chart comparison.

\section{CONCLUSION}

Numerous pharmacological interventions aimed at reducing the overall cardiovascular risk profile have resulted in a significant improvement in the average life expectancy of the population thanks to a reduction in cardiovascular events.

No less important is the role of non-pharmacological measures, among which the correction, through greater health education of the population, of the risk factor "sedentary" represents a low-cost procedure that is easy to implement . Regular and constant exercise helps to achieve well-being and physical fitness (fitness), which are an important indicator of a good general state of health and counteract aging processes.

By physical activity we mean any movement of the body produced by skeletal muscles and involving energy expenditure.

Considering the four cases, there is a rapid improvement of the posture with an implementation of the mobility of the spine and reduction of the algic symptomatology.

Considering the data provided by the Spinal Mouse D360@, the use of isocapnic hyperpnea would be an excellent method for the treatment of spinal paramorphisms, especially of the thoracic segment.

In fact, by modulating the respiratory rate and the volume of the bag, it is possible to intervene on inspiratory musculature retractions, on the hyposthenia of the exhalation musculature and on the hypomobility of the rachis and ribcage, which are the main correlations to the paramorphisms of the rachis. 
In conclusion, the SpiroTiger@ is an excellent tool to associate with custom postural gymnastics protocols to speed up the acquisition of the correct posture.

\section{REFERENCES}

Bordoni B, Marelli F, Bordoni G. A review of analgesic and emotive breathing: a multidisciplinary approach. J Multidiscip Healthc . 2016 Feb; 9: 97-102. https://doi.org/10.2147/jmdh.s101208

Bordoni B, Zanier E. Anatomic Connections of the Diaphragm: Influence of Respiration on the Body System . J Multidiscip Healthc. 2013 Jul.; 6: 281-291. https://doi.org/10.2147/jmdh.s45443

Francavilla VC, Bongiovanni T, Todaro L, Genovesi F, Francavilla G. Risk factors, screening tests and prevention strategies of muscle injuries in èlite soccer players: a critical review of the literature . Med. Sport 2016; Vol. 69:134-50.

Francavilla G, Francavilla VC, Braschi A, Abrignani M, Sciacca R, Caracciolo M, et all. Physical exercise and sport activities in patients with and without coronary heart diseaseischemica. - Esercizio fisico ed attività sportiva in pazienti con e senza cardiopatia ischemica. Monadi Arch Chest Dis 2007; 8795. https://doi.org/10.4081/monaldi.2007.457

Francavilla G, Francavilla VC. Physical exercise is terapy - L'attività motoria è terapia. Med. Sport 2013; Vol.66:625-8.

Francavilla VC, Vitale F, Ciaccio M, Bongiovanni Tindaro, Marotta C, Caldarella R, Todaro L, Zarcone M, Muratore R, Bellia C, Francavilla G, Mazzucco W. Use of Saliva in alternative to serum sampling to monitor biomarkers modifications in professional soccer players. Frontiers in Physiology - 20 December 2018. https://doi.org/10.3389/fphys.2018.01828

Göhl O ,Walker DJ, Walterspacher S, Langer D, Spengler CM, Wanke T, Petrovic M, Zwick RH, Stieglitz S, Glöckl R, Dellweg D, Kabitz HJ. [Respiratory Muscle Training: State of the Art]. Pneumologie. 2016 Jan;70(1):37-48. https://doi.org/10.1055/s-0041-109312

Hodges PW, Butler JE, McKenzie DK, Gandevia SC. Contraction of the human diaphragm during rapid postural adjustments . J Physiol . 1997 Dec 1; 505(Pt 2): 539-548. https://doi.org/10.1111/j.14697793.1997.539bb.x

Hodges PW, Gandevia SC. Activation of the human diaphragm during a repetitive postural task . J Physiol . 2000 Jan 1; 522(Pt 1): 165-175. https://doi.org/10.1111/j.1469-7793.2000.t01-1-00165.xm

Kim MS, Cha YJ, Choi JD. Correlation between forward head posture, respiratory functions, and respiratory accessory muscles in young adults. J Back Musculoskelet Rehabil. 2017 Aug 3;30(4):711-715. https://doi.org/10.3233/bmr-140253

Kocjan J, Adamek M, Gzik-Zroska B, Czyżewski M, Network of Breathing. Multifunctional Role of the Diaphragm: A Review. Adv Respir Med. 2017;85(4):224-232. https://doi.org/10.5603/arm.2017.0037

Szopa A, Domagalska-Szopa M. Correlation between respiratory function and spine and thorax deformity in children with mild scoliosis. Medicine (Baltimore). 2017 Jun;96(22):e7032. https://doi.org/10.1097/md.0000000000007032

Wirth B, Amstalden M, Perk M, Boutellier U, Humphreys BK. Respiratory Dysfunction in Patients With Chronic Neck Pain - Influence of Thoracic Spine and Chest Mobility. Man Ther. 2014 Oct;19(5):4404. https://doi.org/10.1016/j.math.2014.04.011

Wirth B, Ferreira T. Duarte, Mittelholzer M, Humphreys, BK, Boutellier U. Respiratory muscle endurance training reduces chronic neck pain: A pilot study. J Back Musculoskelet Rehabil. 2016 Nov 21;29(4):825-834. https://doi.org/10.3233/bmr-160695

Włodarczyk OM, Barinow-Wojewódzki A. The Impact of Resistance Respiratory Muscle Training With a SpiroTiger® Device on Lung Function, Exercise Performance, and Health-Related Quality of Life in 
Respiratory Diseases. Kardiochir Torakochirurgia Pol. 2015 Dec; 12(4): 386-390. https://doi.org/10.5114/kitp.2015.56796 